\title{
MEASURES WITH SEPARABLE ORBITS
}

\section{R. LARSEN}

1. Introduction. Let $G$ be a locally compact topological (LC) group, and denote by $V(G)$ the linear space of all complex-valued regular Borel measures on $G$ which are finite on compact sets. For $\mu \in V(G)$ and $s \in G$ let $T_{s} \mu$ be that measure in $V(G)$ defined by $T_{s} \mu(E)=\mu(E s)$ for each Borel set $E$ with compact closure. We shall denote by $m$ and $d m$ right invariant Haar measure on $G$, i.e., $T_{s} m=m(s \in G)$; and by $|\mu|$ the total variation of the measure $\mu$. A measure $\mu \in V(G)$ is said to have a separable orbit if there exists a countable subset $C \subset G$ with the property that for each $s \in G$ and $\epsilon>0$ there exists some $c \in C$ such that $\left|T_{s} \mu-T_{c} \mu\right|(G)=\left\|T_{s} \mu-T_{c} \mu\right\| \leqq \epsilon$.

The main result of this paper is the theorem which asserts for second countable LC groups $G$ that if $\mu \in V(G)$ has a separable orbit then $\mu$ is absolutely continuous with respect to $m$. In the case that $|\mu|(G)<\infty$ an immediate consequence of this result is the equivalence of the absolute continuity of $\mu$ and the separability of the orbit of $\mu$.

As usual $M(G)$ will denote the space of $\mu \in V(G)$ for which $|\mu|(G)<\infty$.

\section{Lemmas.}

Lemma 1. Let $G$ be $a$ LC group and $\mu \in V(G)$. For each Borel set $E \subset G$ the complex-valued function $T_{s} \mu(E)$ is Borel measurable.

Proof. Let $\chi_{E}$ denote the characteristic function of the set $E$. Then $\chi_{E}\left(t s^{-1}\right)$ is a Borel measurable function on $G \times G$ and hence $\int_{G} \chi_{E}\left(t s^{-1}\right) d \mu(t)=T_{s} \mu(E)$ is a Borel measurable function on $G$.

Lemma 2. Let $G$ be a second countable LC group, and suppose $\Re$ is the ring of sets generated by some countable basis for $G$ consisting of compact neighborhoods. If $\mu \in V(G)$ and $K \subset G$ is any compact neighborhood then $|\mu|(K)=\sup \sum_{i}\left|\mu\left(L_{i}\right)\right|$ where the supremum is taken over all finite collections of pairwise disjoint sets $L_{i} \in \Re$ whose union is contained in $K$.

Proof. It is evident that $\sup \sum_{i}\left|\mu\left(L_{i}\right)\right| \leqq|\mu|(K)$.

Conversely, suppose $\epsilon>0$ and let $g$ be a bounded Borel measurable function such that $|g|=1$ and $d \mu=g d|\mu|[1$, p. 171]. First, using the regularity of $\mu$, we choose a compact set $C \subset K$ such that $|\mu|(K \sim C)$ $<\epsilon / 3$. Then since $K$ is open we may apply Lusin's theorem [3, p. 53] 1967.

Presented to the Society, November 25, 1966; received by the editors February 6 , 
to find a continuous complex-valued function $f$ with compact support $F$ such that (i) $C \subset F \subset K$, (ii) $\sup |f(t)| \leqq \sup \left|\chi_{C}(t) / g(t)\right|=1$, and (iii) $|\mu|\left(\left\{t \mid f(t) \neq \chi_{C}(t) / g(t)\right\}\right)<\epsilon / 6$. Finally, employing the openness of $K$, the regularity of $\mu$ and a standard construction for the approximation of continuous functions, we construct a function of the form $h=\sum_{i=1}^{n} c_{i} \chi_{L_{i}}$, where $\left|c_{i}\right| \leqq 1, L_{i} \in \Re, i=1,2, \cdots, n, L_{i} \cap L_{j}=\varnothing$, $i \neq j, \cup_{i=1}^{n} L_{i} \subset K$, and such that $\int_{G}|f-h| d|\mu|<\epsilon / 3$.

Combining these inequalities we conclude that,

$$
\begin{gathered}
\left|\int_{G} \chi_{K} d\right| \mu\left|-\int_{G} h g d\right| \mu|| \leqq \int_{G}\left|\chi_{K} / g-h\right| d|\mu| \\
\leqq \\
\quad \int_{G}\left|\chi_{K} / g-\chi_{C} / g\right| d|\mu|+\int_{G}\left|\chi_{C} / g-f\right| d|\mu| \\
\quad+\int_{G}|f-h| d|\mu|<\epsilon / 3+2 \epsilon / 6+\epsilon / 3=\epsilon ;
\end{gathered}
$$

and hence

$$
|\mu|(K)-\epsilon<\left|\int_{G} h g d\right| \mu||=\left|\sum_{i=1}^{n} c_{i} \mu\left(L_{i}\right)\right| \leqq \sum_{i=1}^{n}\left|\mu\left(L_{i}\right)\right| .
$$

Therefore $|\mu|(K)=\sup \sum_{i}\left|\mu\left(L_{i}\right)\right|$.

The third lemma follows immediately from the previous two lemmas.

Lemma 3. Let $G$ be a second countable LC group and $\mu \in V(G)$. For each compact neighborhood $K \subset G$ the real-valued function $\left|T_{s} \mu-\mu\right|(K)$ is Borel measurable.

3. Theorems. To establish the theorem mentioned in the introduction we shall need, besides Lemma 3, one further result which we state without proof. The proof given in $[2$, p. 230] is for abelian groups and finite measures but it is apparent that with some minor modifications of the argument the theorem given here is also valid.

ThEOREM 1. Let $G$ be a LC group and $\mu \in V(G)$. Then the following are equivalent:

(i) $\mu$ is absolutely continuous with respect to $m$.

(ii) For each compact $K \subset G$ the function $T_{s}|\mu|(K)$ is continuous.

The main result of the paper is the following theorem.

THEOREM 2. Let $G$ be a second countable LC group and $\mu \in V(G)$. If $\mu$ has a separable orbit then $\mu$ is absolutely continuous with respect to $m$. 
Proof. We note first that the function $\left\|T_{s} \mu-\mu\right\|$ is a Borel measurable function on $G$. Indeed, since $G$ is $\sigma$-compact there exists a sequence of compact neighborhoods $K_{i}$ such that $K_{i} \subset K_{i+1}$ and $\bigcup_{i=1}^{\infty} K_{i}$ $=G$. The measurability of $\left\|T_{s} \mu-\mu\right\|$ is then a consequence of Lemma 3 and the fact that for each $s \in G \quad\left\|T_{s} \mu-\mu\right\|=\left|T_{s} \mu-\mu\right|(G)$ $=\lim _{n}\left|T_{s} \mu-\mu\right|\left(\bigcup_{i=1}^{n} K_{i}\right)$.

Let $\epsilon>0$ and set $A=\left\{s \mid\left\|T_{8} \mu-\mu\right\| \leqq \epsilon / 2\right\}$. It is evident that $A$ is nonempty, symmetric and, as previously remarked, measurable. Moreover $A$ has positive Haar measure. For $s \in G$ choose $c \in C$ such that $\left\|T_{s} \mu-T_{c} \mu\right\| \leqq \epsilon / 2$. A $c$ satisfying this property exists since the orbit of $\mu$ is separable. However, $\left\|T_{c} c^{-1} s-\mu\right\|=\| T_{c^{-1}} \mu-T_{c}{ }^{-1} c_{c} \mu$ $=\left\|T_{s} \mu-T_{c} \mu\right\| \leqq \epsilon / 2$, and so $c^{-1} s \in A$. Thus $G=\bigcup_{c \in C} c A$, and it follows immediately that $m(A)>0$.

Combining these facts we conclude via a well-known theorem $[1$, p. 296] that $A A$ contains an open neighborhood of the identity in $G$. Furthermore an application of the triangle inequality reveals that $A A \subset\left\{s \mid\left\|T_{s} \mu-\mu\right\| \leqq \epsilon\right\}$, and hence $\left\|T_{s} \mu-\mu\right\|$ is continuous at the identity.

Therefore for each compact $K \subset G$ the function $\left|T_{s} \mu-\mu\right|(K)$, and consequently also the function $T_{s}|\mu|(K)$, is continuous at the identity and thus at all points of $G$.

Finally, an application of Theorem 1 allows us to conclude that $|\mu|$, and so $\mu$, is absolutely continuous with respect to $m$.

For $\mu \in M(G)$ the converse of Theorem 2 can be easily proved.

THEOREM 3. Let $G$ be a second countable LC group and $\mu \in M(G)$. Then the following are equivalent:

(i) $\mu$ is absolutely continuous with respect to $m$.

(ii) $\mu$ has a separable orbit.

Proof. The content of Theorem 2 is that (ii) implies (i).

Suppose $d \mu=f d m$ where $f$ is some Borel measurable function such that $\int_{G}|f| d m<\infty$. Then the separability of the orbit of $\mu$ follows immediately from the continuity of translation of absolutely integrable functions $[1$, p. 285] and the second countability of $G$.

Remarks. (a) If the group $G$ is not second countable then there may exist $\mu \in M(G)$ which is absolutely continuous with respect to $m$ but which does not have a separable orbit. An obvious example is the group $G=R_{d}$, the additive group of the real numbers with the discrete topology, and $\mu=\delta$, the unit mass concentrated at zero.

(b) When $G$ is second countable and $\mu \in V(G) \sim M(G)$ then it is possible for $\mu$ to be absolutely continuous but not have a separable orbit. If $G=R$, the additive group of the real line with the usual topol- 
ogy, then $d \mu(t)=e^{t} d t$ is such a measure. Thus the converse to Theorem 2 is not valid.

(c) On the other hand there do exist absolutely continuous measures $\mu \in V(G) \sim M(G)$, other than complex multiples of Haar measure, which have separable orbits. If we again let $G=R$ then $d \mu(t)=f(t) d t$ where $f(t)=1,|t|<1$, and $f(t)=1 /|t|,|t| \geqq 1$, is one instance of such a measure.

(d) Because of the form in which Theorem 1 is stated here and in [2] it may be of some interest to note an equivalent formulation of the notion of separability for $\sigma$-compact groups, namely: there exists a countable set $C \subset G$ such that for each $s \in G$ and $\epsilon>0$ there exists some $c \in C$ such that $\left|T_{s} \mu-T_{c} \mu\right|(K) \leqq \epsilon$ for all compact sets $K \subset G$.

\section{REFERENCES}

1. E. Hewitt and K. A. Ross, Abstract harmonic analysis, Vol. 1, Academic Press, New York, 1963.

2. W. Rudin, Measure algebras on abelian groups, Bull. Amer. Math. Soc. 65 (1959), 227-247.

3. - Real and complex analysis, McGraw-Hill, New York, 1966.

University of California, Santa Cruz 